\title{
BIOCHAR DE SERRAGEM DE EUCALIPTO COMO CONDICIONADOR DE SUBSTRATOS PARA PRODUÇÃO DE MUDAS DE ALFACE
}

\author{
Luis Fernando Vieira da Silva ${ }^{1}$, Edmar Isaías de Melo², Pablo Arthur Silva Gonçalves ${ }^{3}$
}

\section{RESUMO:}

O sucesso na produção de alface (Lactuca sativa L.) começa pela obtenção de mudas com boa qualidade, sendo necessária a utilização de substratos que forneçam condições químicas e físicas favoráveis ao desenvolvimento da planta. O reaproveitamento de resíduos na produção de substratos pode ser uma proposta interessante na produção de mudas. $\mathrm{O}$ trabalho objetivou avaliar a influência de biochar produzido a partir da serragem de eucalipto, no desenvolvimento de mudas de alface. O experimento foi conduzido em casa de vegetação com delineamento experimental inteiramente casualizado, com sete tratamentos e três repetições. Utilizou-se a cultivar Grandes Lagos e sete substratos sendo um comercial, Bioplant ${ }^{\circledR}$ (BPL); e

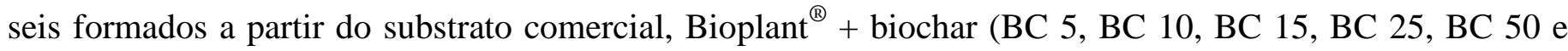
BC $75 \% \mathrm{~m} / \mathrm{m})$. Foram avaliadas as variáveis: percentagem de emergência $(\mathrm{E})$, índice de velocidade de emergência (IVE), comprimento da parte aérea (CPA) e sistema radicular (CSR), massa fresca da parte aérea (MFPA) e sistema radicular (MFSR) e massa seca da parte aérea (MSPA) e sistema radicular (MSSR), 25 dias após a semeadura. A adição de biochar, ao substrato comercial Bioplant ${ }^{\circledR}$, na concentração de $5 \%$ em massa, apresentou viabilidade para ser utilizado como condicionador de substrato no desenvolvimento de mudas de alface da cultivar Grandes Lagos.

Palavras-chave: Desenvolvimento Vegetativo, Lactuca sativa, Olericultura, Pirólise.

\section{EUCALYPTUS SAWDUST BIOCHAR AS A CONDITIONER OF SUBSTRATES FOR PRODUCTION OF LETTUCE SEEDLINGS}

\begin{abstract}
:
The success of lettuce (Lactuca sativa L.) production starts with obtaining good quality seedlings and the use of substrates that provide favorable chemical and physical conditions for plant development. The reuse of residues in substrate production can be an interesting approach for seedling production. The objective of this work was to evaluate the influence of biochar produced from eucalyptus sawdust on the development of lettuce seedlings. The experiment was conducted in a greenhouse using a completely randomized design with seven treatments and three replications. Great Lakes cultivar and seven substrates were used, one commercial, Bioplant ${ }^{\circledR}$ (BPL) and six substrates formed from the commercial substrate,

\footnotetext{
${ }^{1}$ Engenheiro Agrônomo, Mestrando em Agronomia na Universidade Estadual Paulista "Júlio de Mesquita Filho" - UNESP, campus de Jaboticabal, Via de Acesso Professor Paulo Donato Castellane - Vila Industrial, CEP: 14884-900 Jaboticabal (SP), Brasil. luis_fernandosilva2013@hotmail.com

${ }^{2}$ Docente na Universidade Federal de Uberlândia - UFU, campus Monte Carmelo, Rodovia LMG-746, Km 1, CEP: 38500-000 Monte Carmelo (MG), Brasil. emelo@ufu.br

${ }^{3}$ Graduando em Agronomia na Universidade Federal de Uberlândia - UFU, campus Monte Carmelo, Rodovia LMG-746, Km 1, CEP: 38500-000 Monte Carmelo (MG), Brasil. pabloasgoncalves@ gmail.com
} 
Revista Agri-Environmental Sciences, Palmas-TO, v. 5, e019005, 2019

ISSN 2525-4804

Bioplant ${ }^{\circledR}+$ biochar (BC 5, BC 10, BC 15, BC 25, BC50 and BC 75\% w/w). The following variables of emergence percentage (E) were evaluated: emergency speed index (IVE); shoot length (CPA) and root system (CSR); shoot fresh mass (MFPA) and root system (MFSR); and shoot dry mass (MSPA) and root system (MSSR), 25 days after sowing (DAS). The addition of biochar to the commercial substrate, Bioplant ${ }^{\circledR}$, at a concentration of $5 \%$ mass, showed feasibility to be used as a substrate conditioner used in the development of lettuce seedlings of the Great Lakes cultivar.

Keywords: Vegetative Development, Lactuca sativa, Olericulture, Pyrolysis. 


\section{INTRODUÇÃO}

A alface (Lactuca sativa L.) se destaca por ser a hortaliça mais cultivada e consumida no mundo na forma de salada (Ronga et al., 2019; Lopes et al., 2005), apresentando elevada importância socioeconômica, movimentando anualmente, em média, um montante de oito bilhões de reais apenas no varejo, com produção de mais de 1,5 milhões de toneladas ao ano (Abcsem, 2015). A busca dos consumidores por uma alimentação mais saudável, livre de aditivos químicos tem levado produtores de alface de diversos países a utilizar métodos de cultivo que visam práticas produtivas menos dependentes de insumos químicos.

Dentre as diferentes etapas no ciclo de produção de hortaliças, a produção de mudas é considerada uma das principais, pois influência no desempenho final das plantas. $\mathrm{O}$ substrato utilizado, por sua vez, é um dos insumos de extrema importância, devido à influência no ciclo vegetativo e produtivo de mudas e, por consequência, da cultura (Andriolo, 2000). A obtenção de mudas de qualidade necessita de substratos com boa capacidade de retenção de água e aeração, além de proporcionar um meio rico em nutrientes, ser livre de pragas, doenças e propágulos de plantas invasoras (Lei et al., 2017; Mesquita et al., 2012).

Considerando a necessidade de redução nos custos na produção de mudas, a utilização de substratos comerciais condicionados com resíduos da indústria madeireira, por exemplo, a serragem de eucalipto, pode ser uma alternativa viável, além de contribuir para minimizar os impactos ambientais causados pelo descarte incorreto desse resíduo no ambiente. O biocarvão ou "biochar" é obtido através do processo de pirólise, que consiste no aquecimento de biomassa (Rangabhashiyam e Balasubramanian, 2018; Debiagi et al., 2016). O processo de pirólise de materiais lignocelulósicos, representa um benefício ambiental, pois gera produtos cuja composição em termos das substâncias presentes, na sua maioria é orgânica, é um derivado de recursos renováveis, apresenta características que permite sua utilização em sistemas agrícolas de produção (Pandit et al., 2018; Sanchez-Hernandez, 2018; Ding et al., 2017).

O biochar pode ser obtido de resíduos da indústria madeireira, como a serragem de eucalipto. Este material apresenta uma elevada capacidade de retenção de água (C.R.A.) e capacidade de troca de cátions (Menezes Júnior et al., 2000). O processo de pirólise origina um produto (biochar), com maior capacidade de troca de espécies químicas (Li e Chen, 2018; Al-Wabel et al., 2013), além de favorecer a melhoria na estrutura da comunidade microbiana (Du, et al., 2019; Liu et al., 2017).

Diante do exposto, o trabalho foi desenvolvido com objetivo de avaliar o efeito do biochar produzido a partir da serragem de eucalipto, no crescimento de mudas de alface cv. Grandes Lagos, quando adicionado a substratos comerciais.

\section{MATERIAL E MÉTODOS}

O experimento foi conduzido em casa de vegetação da Universidade Federal de Uberlândia, Campus Monte Carmelo - MG (48 43'37', S; 47'31'28' 'W; com altitude de $900 \mathrm{~m}$ ), de novembro a dezembro de 2016. A temperatura da casa de vegetação apresentou uma média mínima de $21^{\circ} \mathrm{C}$ e uma média máxima de $29^{\circ} \mathrm{C}$ durante a realização do experimento.

O delineamento experimental foi inteiramente casualizado com sete tratamentos e três repetições. Os tratamentos corresponderam a sete substratos, sendo o comercial e seis formados a partir da mistura de substrato comercial e biochar, em diferentes concentrações: Bioplant ${ }^{\circledR}$ (BPL), Bioplant $^{\circledR}+$ biochar $5 \%$ (BC 5), Bioplant ${ }^{\circledR}+$ biochar $10 \%$ (BC 10), Bioplant ${ }^{\circledR}+$ biochar $15 \%$ (BC 15), Bioplant ${ }^{\circledR}+$ biochar $25 \%$ (BC 25), Bioplant $^{\circledR}+$ biochar $50 \%$ (BC 50) e Bioplant ${ }^{\circledR}+$ biochar $75 \%$ (BC 75). 
O biochar foi produzido por meio de combustão incompleta pelo processo de pirólise lenta, em forno térmico com dois cilindros adaptado de um modelo amplamente utilizado por agricultores tailandeses (Prakongkep et al., 2015). A fonte de biomassa utilizada para a produção de biochar foi à serragem de eucalipto, resíduo da indústria madeireira. O substrato comercial Bioplant ${ }^{\circledR}$ é composto por fibra de coco, casca de arroz, cinza, esterco e vermiculita, são isentos de microrganismos patogênicos, nematoides e sementes de plantas daninhas.

A semeadura da cultivar Grandes Lagos ( $L$. sativa L.), integrante do tipo de folha americana, foi realizada em bandejas de isopor com 200 células, a $1,0 \mathrm{~cm}$ de profundidade e com três sementes por célula. Aos dez dias após a semeadura (DAS), realizou-se o desbaste mantendo-se a planta mais vigorosa por célula. As bandejas foram colocadas na casa de vegetação, sobre suporte de madeira a uma altura de $1,30 \mathrm{~m}$. A irrigação foi realizada por aspersão uma vez ao dia, complementando com irrigação extra sempre que necessário.

Foram realizadas contagens diárias, computando-se o número de plântulas normais emergidas a cada dia, até que esse permanecesse constante para determinação do índice de velocidade de emergência (IVE) (Equação 1); e no $7^{\circ}$ dia após a semeadura, foi calculado a percentagem de plântulas emergidas (Maguire, 1962).

$$
\mathrm{IVE}=\frac{\mathrm{E}_{1}}{\mathrm{~N}_{1}}+\frac{\mathrm{E}_{2}}{\mathrm{~N}_{2}}+\ldots+\frac{\mathrm{E}_{\mathrm{n}}}{\mathrm{N}_{\mathrm{n}}} \quad \text { Equação (1) }
$$

Sendo: IVE= índice de velocidade de emergência; $\mathrm{E}=$ número de plântulas emergidas computadas a cada contagem; $\mathrm{N}=$ número de dias após a semeadura.

A avaliação do desenvolvimento das plântulas foi realizada 25 dias após a semeadura (DAS), sendo avaliadas quarenta plântulas por parcela totalizando 120 plântulas por tratamento. Avaliou-se o comprimento do sistema radicular (CSR) e comprimento da parte aérea (CPA) determinada a partir da base do caule (coleto) até o ápice da folha mais velha. Em seguida, determinouse a massa fresca da parte aérea (MFPA), massa fresca do sistema radicular (MFSR). Posteriormente, as mudas foram secas em estufa a $60^{\circ} \mathrm{C}$ até massa constante, visando à determinação da massa seca da parte aérea (MSPA) e massa seca do sistema radicular (MSSR).

Os resultados foram submetidos à análise de variância, sendo as médias das variáveis comparadas pelo teste de Tukey a 5\% de probabilidade, utilizando-se o software estatístico R (R Core Team, 2016).

\section{RESULTADOS E DISCUSSÃO}

A percentagem de emergência $(\% \mathrm{E})$ e $\mathrm{o}$ índice de velocidade de emergência (IVE) de plântulas de alface não sofreram influência da presença do biochar, sendo comparável ao substrato comercial (BPL), que resultou em $99,5 \%$ de plântulas emergidas e IVE de 56,6 plântulas dia ${ }^{-1}$ (Tabela 1).

Alterações do solo, ocasionada pela adição do biochar, podem afetar várias propriedades do solo e influenciar, por exemplo, na capacidade de retenção de água, quantidade de água disponível para a planta e permeabilidade de água, que por consequência podem influenciar os parâmetros de germinação, \%E e IVE (Bohara et al., 2019; Norini, et al., 2019).

A não influência da presença do biochar na percentagem de emergência e no índice de velocidade de emergência foram melhores que nos resultados observados no trabalho de Petter (2012), onde a presença do biochar promoveu um efeito de diminuição nestes parâmetros. Dessa forma o biochar utilizado neste trabalho não provocou um desequilíbrio inicial na retenção água-ar e por consequência também não contribuiu para uma maior retenção de umidade nas estruturas porosas do biochar. Esse comportamento também diferiu dos resultados encontrados por Cavalcante et al. (2012), que verificaram um aumento na percentagem de emergência, bem como no índice de velocidade de 
emergência de mudas de maracujazeiro com o aumento da concentração de biochar na composição do substrato.

Tabela 1. Valores médios do IVE e \%E em função da quantidade de biochar no substrato

\begin{tabular}{ccc}
\hline Biochar/\% & \% E & IVE/plântulas \\
\hline BPL & $99,5 \mathrm{a}$ & $56,6 \mathrm{a}$ \\
BC 5 & $99,5 \mathrm{a}$ & $53,4 \mathrm{a}$ \\
BC 10 & $95,0 \mathrm{a}$ & $50,7 \mathrm{a}$ \\
BC 15 & $95,0 \mathrm{a}$ & $52,1 \mathrm{a}$ \\
BC 25 & $96,0 \mathrm{a}$ & $52,3 \mathrm{a}$ \\
BC 50 & $90,5 \mathrm{a}$ & $51,4 \mathrm{a}$ \\
BC 75 & $80,0 \mathrm{a}$ & $48,4 \mathrm{a}$ \\
\hline C.V.\% & 9,31 & 7,49 \\
\hline
\end{tabular}

Médias seguidas de mesma letra minúscula na coluna, não diferem estatisticamente entre si, pelo teste de Tukey a $5 \%$ de probabilidade. IVE = índice de velocidade de emergência e \%E $=$ percentagem de emergência.

Os parâmetros de desenvolvimento da planta, comprimento da parte aérea (CPA), comprimento do sistema radicular (CSR), massa fresca da parte aérea (MFPA), massa fresca do sistema radicular (MFSR), massa seca da parte aérea (MSPA) e massa seca do sistema radicular (MSSR) em função da quantidade de biochar estão apresentados na Tabela 2.

Scotti et al., (2015) relatam que o biochar provoca o aumento do $\mathrm{pH}$, da capacidade de troca de cátions e teor de água, o que por sua vez pode aumentar os nutrientes disponíveis para as plantas e, consequentemente, o acúmulo de biomassa. Esses mesmos autores observaram que a adição de biochar no solo aumentou a atividade de enzimas envolvidas no ciclo de fósforo, nitrogênio e carbono, como as enzimas fosfatase alcalina, fosfatase ácida, fosfohidrolase, lipase esterase, esterase, quimotripsina e tripsina. Pois a presença do biochar no solo pode estimular a comunidades microbianas relacionadas ao nitrogênio, fósforo e ciclagem de carbono (Zhang et al. 2018) e por consequência um maior desenvolvimento da planta.

Tabela 2. Valores médios das variáveis de desenvolvimento de plântulas em função da quantidade de biochar no substrato

\begin{tabular}{ccccccc}
\hline Biochar/\% & MFPA/gGGg & MFSR/g & MSPA/g & MSSR/g & CPA/cm & CSR/cm \\
\hline BPL & $3,05 \mathrm{~b}$ & $3,90 \mathrm{a}$ & $0,85 \mathrm{a}$ & $0,99 \mathrm{a}$ & $2,38 \mathrm{~b}$ & $6,65 \mathrm{a}$ \\
BC 5 & $3,41 \mathrm{a}$ & $4,34 \mathrm{a}$ & $0,89 \mathrm{a}$ & $1,09 \mathrm{a}$ & $2,72 \mathrm{a}$ & $6,86 \mathrm{a}$ \\
BC 10 & $3,08 \mathrm{~b}$ & $3,16 \mathrm{bc}$ & $0,91 \mathrm{a}$ & $0,83 \mathrm{bc}$ & $2,19 \mathrm{c}$ & $6,49 \mathrm{~b}$ \\
BC 15 & $2,48 \mathrm{bc}$ & $2,57 \mathrm{c}$ & $0,84 \mathrm{a}$ & $0,81 \mathrm{bc}$ & $2,16 \mathrm{c}$ & $6,37 \mathrm{~b}$ \\
BC 25 & $2,24 \mathrm{bc}$ & $2,03 \mathrm{c}$ & $0,81 \mathrm{a}$ & $0,75 \mathrm{c}$ & $1,95 \mathrm{~d}$ & $6,36 \mathrm{~b}$ \\
BC 50 & $2,45 \mathrm{bc}$ & $2,40 \mathrm{c}$ & $0,84 \mathrm{a}$ & $0,79 \mathrm{bc}$ & $1,87 \mathrm{~d}$ & $6,35 \mathrm{~b}$ \\
BC 75 & $2,22 \mathrm{c}$ & $2,04 \mathrm{c}$ & $0,82 \mathrm{a}$ & $0,71 \mathrm{c}$ & $1,63 \mathrm{e}$ & $6,45 \mathrm{~b}$ \\
\hline C.V.\% & 13,51 & 17,36 & 11,73 & 11,69 & 15,82 & 13,64 \\
\hline
\end{tabular}

Médias seguidas de mesma letra minúscula na coluna, não diferem estatisticamente entre si, pelo teste de Tukey a $5 \%$ de probabilidade. MFPA = Massa fresca da parte aérea; MFSR= Massa fresca do sistema radicular; MSPA= Massa seca da parte aérea e MSSR= Massa seca do sistema radicular; $\mathrm{CPA}=$ Comprimento da parte aérea; $\mathrm{CSR}=$ Comprimento do sistema radicular.

O substrato com $5 \%$ de biochar (BC 5) promoveu aumento no desenvolvimento vegetativo demonstrando bom desempenho produtivo das plântulas de alface, para as varáveis MFPA e CPA (Tabela 2). O maior crescimento de parte aérea no tratamento BC 5 pode estar relacionado ao fato do biochar contribuir para uma maior absorção de nutrientes do substrato (Bohara et al., 2019) principalmente em função das superfícies reativas nas extremidades das estruturas aromáticas nos poros do biochar (Petter, 2012).

Com relação às demais variáveis (MFSR, MSPA, MSSR e CSR) o substrato contendo $5 \%$ de biochar não apresentou diferenças, pelo teste de Tukey ao nível de significância de $5 \%$ em relação ao substrato Bioplant ${ }^{\circledR}$. No entanto, em concentrações 
acima de BC 5 de biochar, verificou-se redução em todas as variáveis de desenvolvimento de plântulas avaliadas. Esse efeito negativo pode estar associado a um desequilíbrio nutricional ocasionado pelo excesso de matéria orgânica ou pelo fato do biochar ter modificado a quantidade de microporos considerada adequada para produção de hortaliças, o que conferiu aos substratos BC 10 à BC 75 de uma capacidade de retenção de água insatisfatória, influenciando negativamente o sistema radicular (Santos et al., 2010).

Esse comportamento para as variáveis MFSR, MSPA, MSSR e CSR, para concentrações de biochar maiores que $5 \%$, corroboram com os resultados encontrados por Petter (2012), que relatou que concentrações de biochar superiores a 7,5\% influenciaram negativamente, principalmente o desenvolvimento radicular. Já a massa seca da parte aérea (Tabela 2), não foi influenciada pelos diferentes tratamentos, corroborando com os resultados encontrados por Lopes et al. (2007), que não verificaram nenhuma diferença entre os tratamentos para massa seca das plântulas de alface.

O maior valor de comprimento da parte aérea (CPA) para o tratamento $\mathrm{BC} 5$, pode estar relacionado ao fato do biochar ter contribuído para uma maior absorção de nutrientes, principalmente em função dos grupos funcionais reativos, presentes em estruturas moleculares aromáticas do biochar (Ghezzehei et al., 2014; De Figueiredo et al., 2017; Silva et al., 2017).

Observou-se que em concentrações maiores que $5 \%$ de biochar promoveram uma redução no crescimento da parte aérea e do sistema radicular, o que pode ser atribuído a compostos fenólicos ou poliaromáticos (Bueno, 2017), adicionados pelo biochar ao substrato o que pode representar um aumento de fitoxicidade e prejudicar o desenvolvimento da plântula. Ou mesmo a substituição gradual do substrato comercial Bioplant $^{\circledR}$, também pode ter corroborado para redução das concentrações de nutrientes presentes no substrato final.

\section{CONCLUSÃO}

A adição de biochar, produzido a partir serragem de eucalipto e adicionado ao substrato comercial Bioplant $^{\circledR}$, na concentração de 5,0\% em massa, apresentou viabilidade para ser utilizado como condicionador de substrato, sendo que concentrações acima deste valor não contribuiu para o desenvolvimento de mudas de alface da cultivar Grandes Lagos.

\section{AGRADECIMENTOS}

Os autores agradecem à Fundação de Amparo à Pesquisa do estado de Minas Gerais (FAPEMIG) e ao Conselho Nacional de Desenvolvimento Científico e Tecnológico (CNPq) o apoio financeiro para a condução dessa pesquisa.

\section{REFERÊNCIAS BIBLIOGRÁFICAS}

ABCSEM - Associação Brasileira do Comércio de Sementes e Mudas. (2015). Alface é a folhosa mais consumida no Brasil. Disponível em: http://www.grupocultivar.com.br/noticias/alface-e-afolhosa-mais-consumida-no-brasil

Al-Wabel, M.I.; Al-Omaran, A.; El-Naggar, A.; Nadeem, M.; \& Usman, A.R.A. (2013). Pyrolysis temperature induced changes in characteristics and chemical composition of biochar produced from conocarpus wastes. Bioresource Technology 131(1): 374-379.

https://doi.org/10.1016/j.biortech.2012.12.165

Andriolo, J. L. (2000). Fisiologia da produção de hortaliça em ambiente protegido. Horticultura Brasileira 18(1): 26-32.

Bohara, H.; Dodla, S.; Wang, J.J.; Darapuneni, M.; Acharya, B.S.; Magdi, S.; \& Pavuluri, K. (2019). Influence of poultry litter and biochar on soil water dynamics and nutrient leaching from a very fine sandy loam soil. Soil \& Tillage Research 189(1): 44-51. https://doi.org/10.1016/j.still.2019.01.001 
Bueno, C.C. (2017). Biochar: caracterização estrutural e interações com nutrientes e microrganismos pedológicos. (Tese de doutorado). Universidade Estadual Paulista "Júlio de Mesquita Filho". Sorocaba, SP, Brasil p. 158.

Cavalcante, I.H.L.; Petter, F.A.; Albano, F.G.; Silva, R.R.S.; \& Júnior, G.B.S. (2012) Biochar no substrato para produção de mudas de maracujazeiro amarelo. Revista de La Facultad de Agronomia 111(1): 41-47.

De Figueiredo, N.A.; Costa, L.M.; Melo, L.C.A.; Siebeneichlerd, E.A.; \& Tronto, J. (2017). Characterization of biochars from different sources and evaluation of release of nutrients and contaminants. Revista Ciência Agronômica 48(3): 395-403. https://doi.org/10.5935/1806$\underline{6690.20170046}$

Debiagi, P.E.A.; Gentile, G.; Pelucchi, M.; Frassoldati, A.; Cuoci, A.; Faravelli, T.; Ranzi, E. (2016). Detailed kinetic mechanism of gas-phase reactions of volatiles released from biomass pyrolysis. Biomass and Bioenergy 93(1): 60-71. https://doi.org/10.1016/j.biombioe.2016.06.015

Ding, Y.; Liu, Y.; Liu, S.; Huang, X.; Li, Z.; Tan, X.; Zeng, G.; \& Zhou, L. (2017). Potential Benefits of Biochar in Agricultural Soils: A Review. Pedosphere 27(4): 645-661. https://doi.org/10.1016/S1002-0160(17)60375-8

Du, J.; Zhang, Y.; Qu, M.; Yin, Y.; Fan, K.; Hu, B.; Zhang, H.; Wei, M.; \& Ma, C. (2019). Effects of biochar on the microbial activity and community structure during sewage sludge composting. Bioresource Technology 272(1): 171-179. https://doi.org/10.1016/j.biortech.2018.10.020

Ghezzehei, T.A.; Sarkhot, D.V.; \& Berhe, A.A. (2014) Biochar can be used to capture essential nutrients from dairy wastewater and improve soil physico-chemical properties. Solid Earth 5(2): 953962.. https://doi.org/10.5194/se-5-953-2014

Lei, W.; Ding, Y.; Li, G.; Tang, S.; \& Wang, S. (2017). Effects of soilless substrates on seedling quality and the growth of transplanted super japonica rice. Journal of integrative agriculture
16(5): 1053-1063. https://doi.org/10.1016/S2095$\underline{3119(16) 61588-5}$

Li, S.; Chen, G. (2018). Thermogravimetric, thermochemical, and infrared spectral characterization of feedstocks and biochar derived at different pyrolysis temperatures. Waste Management $78(1)$ 198-207. https://doi.org/10.1016/j.wasman.2018.05.048

Liu, N.; Zhou, J.; Han, L.; Ma, S.; Sun, X.; \& Huang, G. (2017). Role and multi-scale characterization of bamboo biochar during poultry manure aerobic composting. Bioresource Technology 241(1): 190-199. https://doi.org/10.1016/j.biortech.2017.03.144

Lopes, J.C.; Ribeiro, L.G.; Araújo, M.G.; \& Beraldo, M.R.B.S. (2005). Lettuce production using doses of sewage sludge. Horticultura Brasileira 23(1): 143147.

$\underline{05362005000100030}$

Lopes, J.L.W.; Boaro, C.S.F.; Peres, M.R.; \& Guimarães, V.F. (2007). Crescimento de mudas de alface em diferentes substratos. Biotemas 20(4): 1925. https://doi.org/10.5007/\%25x

Maguire, J. D. (1962). Speed of germination-aid selection and evaluation for seedling emergence and vigor. Crop Science 2(2): 176-177. https://doi.org/10.2135/cropsci1962.0011183X0002 $\underline{00020033 x}$

Menezes Júnior, F.O.G.; Fernandes, H.S.; Rogério, M.C.; \& Silva, J.B. (2000). Caracterização de diferentes substratos e seu desempenho na produção de mudas de alface em ambiente protegido. Horticultura Brasileira 18(3): 164-170. https://doi.org/10.1590/S0102-05362000000300004

Mesquita, E.F.; Chaves, L.H.G.; Freitas, B.V.; Silva, G.A.; Sousa, M.V.R.; \& Andrade, R. (2012). Produção de mudas de mamoeiro em função de substratos contendo esterco bovino e volumes de recipientes. Revista Brasileira de Ciências Agrárias 7(1): 58-65. https://doi:10.5039/agraria.vti1a1448

Norini, M.P.; Thouin, H.; Miard, F.; Battaglia, F.; Gautret, P.; Guégan, R.; Le F.L.; Morabito, D.; Bourgerie, S.; \& Motelica, M. (2019). Mobility of 
$\mathrm{Pb}, \mathrm{Zn}, \mathrm{Ba}, \mathrm{As}$ and $\mathrm{Cd}$ toward soil pore water and plants (willow and ryegrass) from a mine soil amended with biochar. Journal of Environmental Management 232(1): 117-130. https://doi10.1016/j.jenvman.2018.11.021

Pandit, N.R.; Mulder, J.; Hale, S.R.; Martinsen, V.; Schmidt, H.P.; \& Cornelissen, G. (2018). Biochar improves maize growth by alleviation of nutrient stress in a moderately acidic low-input Nepalese soil. Science of The Total Environment 625(1): 1380-1389.

https://doi.org/10.1016/j.scitotenv.2018.01.022

Petter, F.A.; Júnior, B.H.M.; Andrade, F.R.; Schossler, T.R.; Gonçalves, L.G.; \& Marimon, B.S. (2012). Biochar como condicionador de substrato para a produção de mudas de alface. Agrarian 5(17): 243-250.

Prakongkep, N.; Gilkes, R.J.; \& Wiriyakitnateekul, W. (2015). Forms and solubility of plant nutrient elements in tropical plant waste biochars. Journal of Plant Nutrition and Soil Science 178(5): 732-740. https://doi.org/10.1002/jpln.201500001

\section{R CORE TEAM. R: A language and environment} for statistical computing. (2016). R Foundation for Statistical Computing, Vienna, Austria. Disponível em: https://www.r-project.org/

Rangabhashiyam, S.; Balasubramanian, P. (2018). The potential of lignocellulosic biomass precursors for biochar production: Performance, mechanism and wastewater application-A review. Industrial Crops \& products 128(1): 405-423. https://doi.org/10.1016/j.indcrop.2018.11.041

Ronga, D.; Setti, L.; Salvarani, C.; Leo, R.; Bedin, E.; Pulvirenti, A.; Milc, J.; Pecchioni, N.; \& Francia, E. (2019). Effects of solid and liquid digestate for hydroponic baby leaf lettuce (Lactuca sativa L.) cultivation. Scientia Horticulturae 244(1): 172181. https://doi.org/10.1016/j.scienta.2018.09.037

Sanchez-Hernandez, J. C. (2018). Biochar activation with exoenzymes induced by earthworms: A novel functional strategy for soil quality promotion. Journal of Hazardous Materials 350(1): 136-143. https://doi:10.1016/j.jhazmat.2018.02.019
Santos, M.R.; Sediyama, M.A.N.; Salgado, L.T.; Vidigal, S.M.; \& Reigado, F.R. (2010). Produção de mudas de pimentão em substratos à base de vermicomposto. Bioscience Journal 26(4): 572578.

Scotti, R.; Bonanomi, G.; Scelza, R.; Zoina, A.; \& Rao, M.A. (2015). Organic amendments as sustainable tool to recovery fertility in intensive agricultural systems. Journal of Soil Science and Plant Nutrition 15(2): 333-352. https://doi.org/10.4067/S0718-95162015005000031

Silva, I.C.B.; Fernandes, L.A.; Colen, F.; \& Sampaio, R.A. (2017). Growth and production of common bean fertilized with biochar. Ciência Rural 47(11): $\quad 1-8 . \quad$ https://doi.org/10.1590/0103$\underline{8478 \mathrm{cr} 20170220}$

Zhang, G.; Guo, X.; Zhu, Y.; Liu, X.; Han, Z.; Sun, K.; Ji, L.; He, Q.; \& Han, L. (2018). The effects of different biochars on microbial quantity, microbial community shift, enzyme activity, and biodegradation of polycyclic aromatic hydrocarbons in soil. Geoderma 328(1): 100-108. https://doi.org/10.1016/j.geoderma.2018.05.009 